\title{
CP-CRE/non-CP-CRE Stratification And CRE
}

\section{Resistance Mechanism Determination Help In Better Managing CRE Bacteremia Using Ceftazidime- Avibactam And Aztreonam-Avibactam}

This article was published in the following Dove Press journal:

Infection and Drug Resistance

Hua Zou',*

Sen-jie Xiong ${ }^{2} * *$

Qiu-Xia Lin ${ }^{1, *}$

Meng-Lu Wu'

Si-Qiang Niu (iD)

Shi-Feng Huang $\mathbb{D}^{\prime}$

'Department of Laboratory Medicine, The First Affiliated Hospital of Chongqing Medical University, Chongqing 400016,

People's Republic of China; ${ }^{2}$ Department of Neurosurgery, The First Affiliated

Hospital of Chongqing Medical University, Chongqing 400016, People's Republic of

China

*These authors contributed equally to this work
Correspondence: Si-Qiang Niu;

Shifeng Huang

Department of Laboratory Medicine,

The First Affiliated Hospital of Chongqing Medical University, No. I Friendship Road, Yuzhong District, Chongqing 4000I6,

People's Republic of China

Tel +86 23890 I 2742;

Tel +86 I86 23027077

Fax +862389012513

Email siqiangniu@cqmu.edu.cn;

shuang@hospital.cqmu.edu.cn
Purpose: This observational study aimed to identify the independent risk factors for both the acquisition and mortality of carbapenemase-producing carbapenem-resistant Enterobacteriaceae (CP-CRE) bacteremia and further assess the in vitro antimicrobial activities of ceftazidimeavibactam (CAZ/AVI) and aztreonam-avibactam (ATM/AVI) against recent CRE bacteremic isolates.

Patients and methods: This observational study was conducted to reveal the risk factors and mortality rate for CP-CRE bacteremia between 2012 and 2018 and also evaluate the in vitro antimicrobial activities of CAZ/AVI and ATM/AVI against recent CRE bacteremic isolates from 2016 to 2018.

Results: A total of 81 non-repetitive isolates were collected from 2012 to 2018 , with $67.90 \%$ (55/81) being CP-CRE. Old age $(P=0.01)$, transfusion [odds ratio (OR): 17.19; 95\% CI: 3.1593.72; $P=0.001$ ], longer ICU stay $(P=0.02)$, cancer (OR: $15.91 ; 95 \%$ CI: $3.56-71.37 ; P<$ 0.001 ), and previous carbapenem exposure (OR: 27.86; 95\% CI: $5.03-154.19 ; P=0.001)$ were identified as independent risk factors for the acquisition of CP-CRE bacteremia compared with the ESBL bacteremia. The in vitro antimicrobial activities of CAZ/AVI and ATM/AVI against the CRE bacteremic isolates from 2016 to 2018 showed a respective susceptibility rate of $70.68 \%$ $(41 / 58)$ and $100.00 \%(58 / 58)$.

Conclusion: The findings indicated that both CP-CRE/non-CP-CRE stratification and CRE resistance mechanism determination were necessary for better guiding the clinical management of CRE bacteremia: ATM/AVI probably works with both non-CP-CRE and CP-CRE bacteremia, even the most notorious double-carbapenemase producer with porin loss/deficiency, whereas CAZ/AVI works with most of the non-CP-CRE and KPC-producers in the region.

Keywords: aztreonam-avibactam, carbapenemase-producing carbapenem-resistant Enterobacteriaceae, carbapenem-resistant Enterobacteriaceae bacteremia, ceftazidime-avibactam

\section{Introduction}

Carbapenem-resistant Enterobacteriaceae (CRE) has become a serious public health threat worldwide. ${ }^{1,2}$ Severe CRE infections, especially CRE bacteremia, are associated with an extremely high mortality rate of up to $70 \%,{ }^{3}$ thus, timely, efficient, and targeted antibiotic treatment is of paramount importance. ${ }^{4}$

$\mathrm{CRE}$ arises from one or a combination of the following four mechanisms: carbapenemase production (CP-CRE), production of ESBLs and/or AmpC in 
combination with porin loss/deficiency (non-CP-CRE), carbapenem efflux, or mutations in penicillin-binding proteins (PBPs), ${ }^{5}$ among which CP-CRE is the most problematic due to higher-level antimicrobial resistance and plasmid localization of many carbapenemase-encoding genes, potentiating the possibility of horizontal gene transfer. ${ }^{6}$

Carbapenemases include Ambler class A $\beta$-lactamases (e.g., KPC and GES), class B metallo- $\beta$-lactamases (MBLs; e.g., NDM, VIM, IMP, and SPM), and class D $\beta$-lactamases (e.g., OXA-48 and OXA-181). ${ }^{5}$ MBLs are particularly worrisome due to their ability to hydrolyze all classes of $\beta$ lactams except monobactams (aztreonam) and the inability of the classic serine $\beta$-lactamase inhibitors (clavulanic acid, tazobactam, and sulbactam) to inhibit them. On the contrary, the availability of more reliable phenotypic and genotypic carbapenemase assays has led to a better understanding of the implications of resistance heterogeneity in the clinical management of severe CRE infections. ${ }^{6}$

In the last decade, with polymyxins, tigecycline, fosfomycin, and aminoglycosides being the therapeutic mainstays for CRE infections, double carbapenem, high-dose prolonged carbapenem infusion, and high-dose tigecycline therapies as the combination therapeutic strategies and therapeutic regimens were mainly experience-directed. ${ }^{1,7}$ With the advent of the newly additional effective therapeutic options for CRE infections, such as the novel $\beta$ lactam/ $\beta$-lactamase inhibitors aztreonam/avibactam (ATM/ AVI, active against KPC, MBL, AmpC and OXA producers), ${ }^{8}$ ceftazidime/avibactam (CAZ/AVI, active against KPC and OXA-48 producers), ${ }^{9}$ meropenem/vaborbactam (active against KPC producers), ${ }^{10}$ imipenem/relebactam (active against KPC and AmpC producers), ${ }^{11}$ and the newly developed antibiotics eravacycline (a novel tetracycline derivative), ${ }^{12}$ plazomicin (a next-generation aminoglycoside), ${ }^{13}$ and cefiderocol (a novel siderophore cephalosporin), ${ }^{14}$ the therapeutic recipe might be targeted and personalized based on the antimicrobial susceptibility profiles, molecular resistance phenotypes, disease severity, and patient characteristics. By prioritizing who should receive novel $\mathrm{CP}-\mathrm{CRE}$ active antibiotic agents, treatment success can be maximized and drug resistance reduced. More high-quality epidemiology- and resistance mechanism-centered studies are urgently needed to guide effective individualized and targeted therapy for CRE bacteremia. Nevertheless, previous studies exploring the prognosis of CRE bacteremia simply evaluated CRE bloodstream infections as a single cohort, without discriminating between the underlying molecular resistance mechanisms. To date, only one study compared the clinical outcomes of CP-CRE and non-CP-CRE bacteremia by examining $83 \mathrm{CRE}$ bacteremia cases from the Johns Hopkins Hospital between March 2013 and April 2016. It demonstrated that CP-CRE was associated with poorer outcomes, suggesting that CP-CRE might be more virulent compared with non-CP-CRE, highlighting the added significance of deciphering the underlying molecular resistance mechanisms of CRE to direct the personalized antibiotic therapy for CRE bacteremia. ${ }^{15}$ Thus, for better guidance of the clinical management of CRE bacteremia, additional epidemiology and resistance testing of bacteremia CRE isolates from other countries or regions worldwide are urgently needed.

Avibactam (AVI) offers a broader $\beta$-lactamase inhibition profile compared with any other recently used serine $\beta$-lactamase inhibitors. ${ }^{16}$ When combined with AVI, CAZ/ AVI is active in vitro against CP-CRE and non-CP-CRE isolates. ${ }^{17}$ Notably, recent studies have demonstrated its potent in vitro bactericidal activity against $b l a_{\mathrm{KPC}^{-}}$and bla $_{\text {OXA-48-harboring CP-CRE isolates. }}{ }^{11}$

Although aztreonam (ATM) is stable to MBL hydrolysis, it is easily inactivated by ESBLs, KPCs, and AmpC. ${ }^{18}$ As MBLs-harboring Enterobacteriaceae may frequently harbor additional ATM-inactivating $\beta$-lactamases, the bactericidal activity of ATM against these isolates is often mitigated or negated. When combined with AVI, ATM/AVI can kill KPC, AmpC, MBL, and OXA producers. $^{18}$

CAZ/AVI and ATM/AVI have been used to treat severe cases of CRE infections in the United States, and the resistance had sporadically been reported. ${ }^{19,20}$ This observational study was conducted to identify the independent risk factors for both the acquisition and mortality of CP-CRE bacteremia and further assess the in vitro antimicrobial activities of CAZ/AVI and ATM/AVI against recent CRE bacteremic isolates.

This study was the first systemic investigation of the possible value of CP-CRE/non-CP-CRE stratification and CRE resistance mechanism determination in better managing CRE bacteremia by both CAZ/AVI and ATM/AVI.

\section{Materials And Methods Bacterial Strains}

A total of 81 non-repetitive nosocomial CRE bacteremic strains were collected between 2012 and 2018 in the First Affiliated Hospital of Chongqing Medical University. All 
the isolates were identified at the species level by the VITEK MS (bioMérieux, MO, USA) system, and routine antimicrobial susceptibility testing was performed using the VITEK2 compact (bioMérieux, Inc., NC, USA) system. All the bacteremia CRE mono-infection cases with intact medical records and available CRE isolates were included in the study.

\section{Resistance Mechanism Identification}

Polymerase chain reaction was used to detect the potential presence of resistance genes, including carbapenemases $\left(b l a_{\mathrm{KPC}}, b l a_{\mathrm{NDM}}, b l a_{\mathrm{VIM}}, b l a_{\mathrm{IMP}}, b l a_{\mathrm{GES}}, b l a_{\mathrm{OXA}-48-\mathrm{like}}\right.$, $b l a_{\text {OXA-181-like, }} b l a_{\text {OXA-23-like, }} b l a_{\text {OXA-24-like, and }} b l a_{\text {OXA- }}$ 58-like), ${ }^{21}$ ESBLs, AmpC, Ompk35, Ompk36, OmpF, and OmpC genes, using primers as described previously. ${ }^{22}$ In addition, the capsular genes of Klebsiella pneumoniae were also amplified. The Carba NP test and eCIM were performed on all isolates to determine whether any bacteria produced carbapenemases by phenotypic methods but were negative by genotypic methods, or vice versa. ${ }^{21}$

\section{Risk Factors And Clinical Outcomes Of Patients With Bacteremia CP-CRE}

This retrospective case-control study was conducted to evaluate the risk factors and clinical outcomes of the patients suffering from CP-CRE bacteremia. All patients with CP-CRE bacteremia were selected as cases. Controls were identified as patients with non-CP-CRE bacteremia or ESBLs-positive Enterobacteriaceae bacteremia.

\section{Antibiotics And In Vitro Susceptibility Testing}

CRE bacteremic strains from 2016 to 2018 were recovered for CAZ/AVI and ATM/AVI susceptibility tests. The broth microdilution test method was employed to determine the MICs of CAZ, CAZ/AVI, ATM, and ATM/AVI according to CLSI 2018. MICs of CAZ/AVI $>16 / 4 \mu \mathrm{g} / \mathrm{mL}$ and ATM/ AVI $>16 / 4 \mu \mathrm{g} / \mathrm{mL}$ were considered resistant.

\section{Statistical Analysis}

All analyses were performed using the SPSS v.25.0 software (SPSS Inc., IL, USA). Univariate analyses were performed separately for each of the variables. All variables with a $P$ value of $\leq 0.05$ in the univariate analyses were considered for inclusion in the multivariate logistic regression model. The odds ratio (OR) and 95\% confidence interval $(\mathrm{CI})$ were calculated to evaluate the strength of any association. For all calculations, statistical significance was defined at $P<0.05$ for two-tailed tests.

\section{Ethical Considerations}

The data and samples analyzed in the present study were obtained in accordance with the standards and approved by the Chongqing Medical University Institutional Review Board and the Biomedical Ethics Committee. For this study, samples were collected at the microbiology laboratory of the hospital, with no contact with the patient. This study was retrospective with no patient identification performed during data collection. Therefore, the ethics committee determined that informed consent was not required.

\section{Results}

\section{Microbiological Characteristics And Antimicrobial Susceptibility Profiles Of CRE Bacteremic Isolates}

As shown in Figure 1, 81 non-repetitive and mono-infected CRE bacteremic isolates were collected during the study period, among which the predominant genus and species were $K$. pneumoniae (56.79\%), followed by Enterobacter cloacae (22.22\%) and Escherichia coli (18.52\%) (Figure 1A). Resistance mechanism determination revealed that $67.90 \%$ of the isolates produced carbapenemases and the proportion of CP-CRE increased every year (Figure 1B). Among all the CP-CRE isolates, $K$. pneumoniae $(65.45 \%)$ was the most common species, with E. coli and E. cloacae accounting for $20 \%$ and $9.09 \%$, respectively (Figure 1C). It was speculated that the bacteremic CRE isolate might have derived from the tissue origins from which the same-species CRE isolate with the same antibiogram was isolated. As the most common specimen types from which the same-species-same-antibiogram isolate was derived were sputum (50.61\%), urine (28.39\%), and bile (12.34\%) (Figure 1D), it was deduced that the most dominant origins of the CRE bacteremia were respiratory, urinary, and biliary systems.

Regarding the antimicrobial susceptibility profiles of the CRE bacteremic isolates (Table 1), CRE showed the highest nonsusceptibility rate to ertapenem (100.00\%), with $77.78 \%$ and $65.43 \%$ nonsusceptibility rates to imipenem and meropenem, respectively. Although these isolates also exhibited high resistance rates to quinolones $(>80 \%)$ and aminoglycosides $(>70 \%)$, they showed low resistance rates to tigecycline $(2.46 \%)$ and colistin $(3.70 \%)$. 
A)

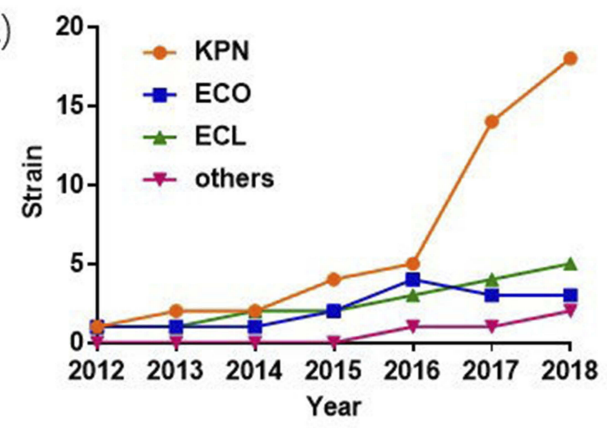

C)

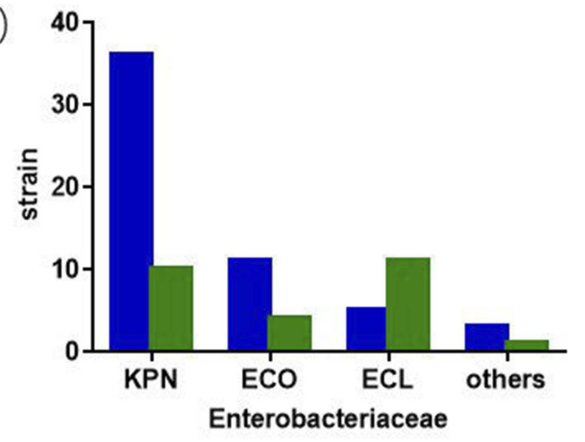

B)

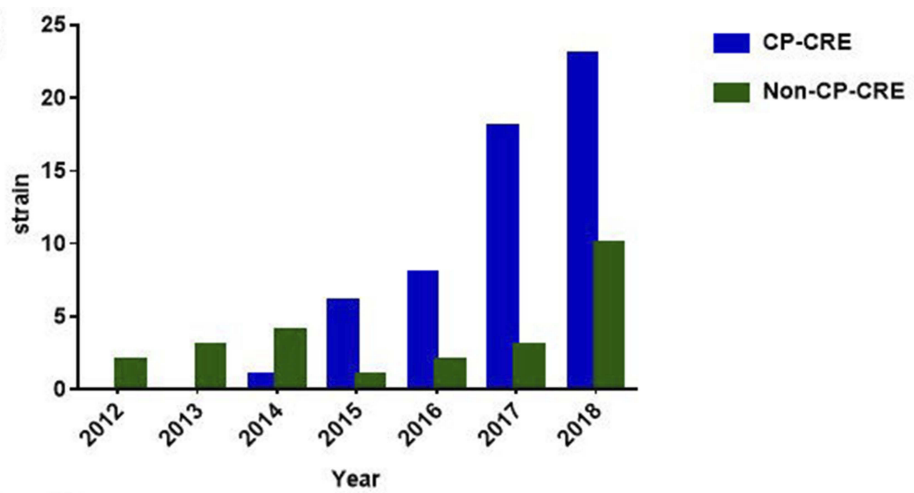

D)

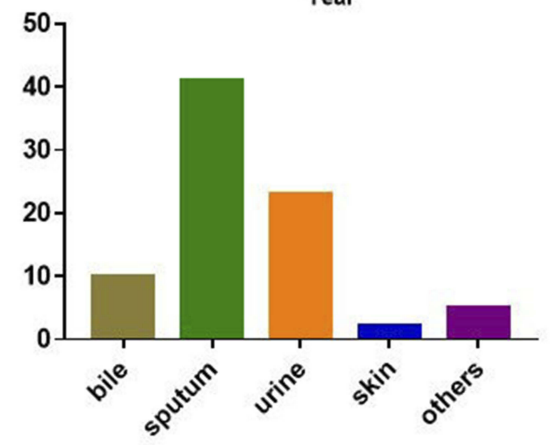

Figure I Distribution of CRE bacteremia cases and bacteremic isolates. (A) Dynamic distribution of species-specific CRE bacteremic isolates. (B) Dynamic distribution of CP-CRE and non-CP-CRE bacteremia cases. (C) Species distribution of CP-CRE and non-CP-CRE bacteremic isolates. (D) Source of CRE bacteremia.

\section{Risk Factors And Clinical Outcomes Of Patients With Bacteremia CRE}

To investigate the risk factors and prognosis of patients with CP-CRE bacteremia, 55 CP-CRE cases were matched to either 25 non-CP-CRE cases or 60 ESBLs cases in this study where appropriate. The results (Table 2) showed a 50\% (40/80) mortality rate among all the patients with CRE bacteremia. However, when stratified into CP-CRE and non-CP-CRE subgroups, the mortality rate was $61.82 \%$ and $24 \%$, respectively, showing a significantly higher mortality rate in the CP-CRE subgroup. Moreover, patients with CP-CRE bacteremia showed longer hospital stay. Notably, the CP-CRE, non-CPCRE, and ESBL groups were well balanced on most of the demographic information, pre-existing medical conditions, and immune-compromising comorbidities, and a likely source of bacteremia (Table 2). Old age $(P=0.04)$, longer ICU stay $(P=0.03$ ), cancer (OR: 4.35 ; 95\% CI: $1.22-15.46 ; P=0.023$ ), and previous carbapenem exposure (OR: $7.67,95 \%$ CI: $1.91-$ $30.77, P=0.004)$ were identified as independent risk factors for the acquisition of CP-CRE bacteremia compared with nonCP-CRE bacteremia. Old age $(P=0.01)$, transfusion (OR: 17.19; 95\% CI: 3.15-93.72; $P=0.001)$, longer ICU stay $(P=0.02)$, cancer (OR: 15.91; 95\% CI: 3.56-71.37; $P<0.001$ ), and previous carbapenem exposure (OR: 27.86; 95\% CI: $5.03-154.19 ; \quad P=0.001)$ were identified as independent risk factors for the acquisition of CP-CRE bacteremia compared with the ESBL bacteremia (Table 3). On the other hand, longer ICU stay $(P=0.018)$ and venous catheterization (OR: 10.29 ; 95\% CI: 3.03-34.87; $P=0.001$ ) were identified as independent risk factors for non-CP-CRE bacteremia compared with the ESBL bacteremia group.

\section{Molecular Analysis Of Carbapenem Resistance Mechanisms}

As shown in Table 4, the most common carbapenemase genes were KPC (63.64\%, 35/55), NDM (32.73\%, 18/55), and IMP $(5.45 \%, 3 / 55)$. Notably, a K. pneumoniae isolate that simultaneously expressed KPC-2 and IMP-4 with porin deficiency was identified. All the MBLs-producing (NDM-1, NDM-5, IMP-4, and IMP-8) strains were resistant to all the carbapenem antibiotics tested. For non-CP-CRE bacteremic isolates, ESBL overexpression with OMP loss/deficiency was found to be the most predominant resistance mechanism, accounting for $88.46 \%(23 / 26)$.

\section{Bactericidal Activities Of CAZ/AVI And ATM/AVI Against CRE Bacteremic Isolates}

To better define the antimicrobial profiles of CAZ/AVI and ATM/AVI against the CRE bacteremic isolates, a recent (2016-2018) collection of 58 non-repetitive CRE bacteremic 


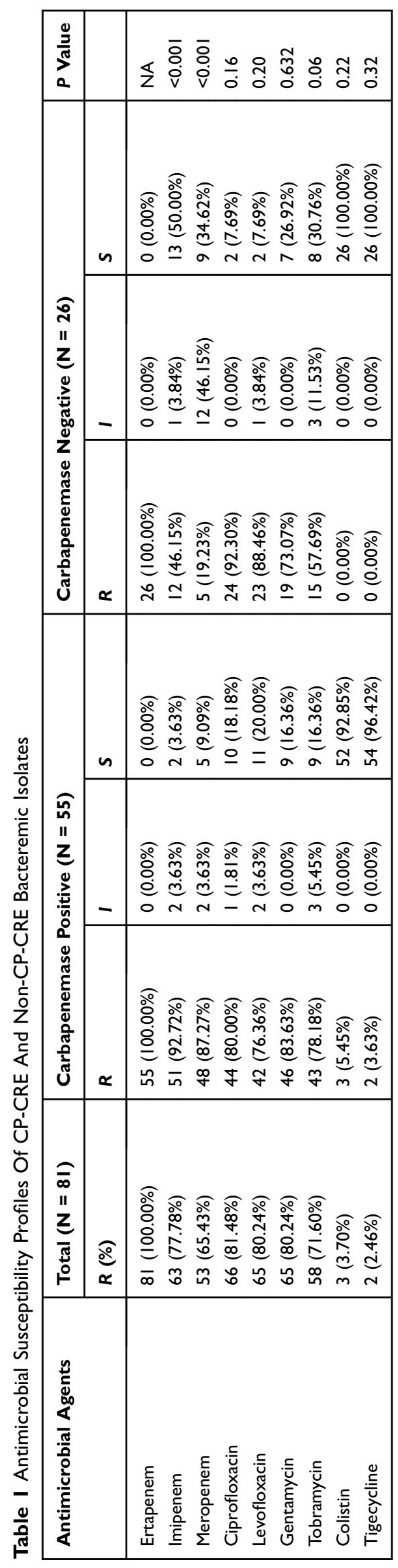

isolates, including $44 \mathrm{CP}-\mathrm{CRE}$ strains and 14 non-CP-CRE strains, was generated. The in vitro antimicrobial susceptibilities of CAZ, CAZ/AVI, ATM, and ATM/AVI against these isolates were determined using the CLSI broth microdilution method.

As was shown in Table 5, CAZ/AVI could inhibit most of the non-CP-CRE isolates $(85.71 \%, 12 / 14)$, with low-level resistance (MIC: $16 / 4 \mu \mathrm{g} / \mathrm{mL}$ ) in only two strains. For CPCRE isolates, most strains carrying the KPC gene could be inhibited by CAZ/AVI $(93.55 \%, 29 / 31)$. However, when an isolate expressed class B enzymes (such as NDM and IMP), CAZ/AVI could not inhibit its growth anymore. Compared with CAZ/AVI, ATM/AVI exhibited superior bactericidal activity, inhibiting the growth of all CRE strains.

\section{Discussion}

CRE bacteremia was previously reported to be associated with extremely high mortality, ranging from $20 \%$ to $70 \%{ }^{23,24}$ This cohort study showed a 50\% (40/80) mortality rate in general. However, when stratified into CP-CRE and non-CP-CRE groups, the mortality rate of the CP-CRE subgroup (61.82\%) was significantly higher than that of the non-CP-CRE group (24\%), supporting earlier observations that CP-CRE bacteremia was more harmful than non-CP-CRE bacteremia, with higher levels of antimicrobial resistance and greater mortality. ${ }^{15}$ Therefore, for the effectiveness of the treatment and prevention of antibiotic resistance, the antibiotics and doses used to treat CP-CRE and non-CP-CRE bacteremia should be different. Of note, CarbaNP and mCIM with or without eCIM were currently recommended by CLSI to be performed on CRE isolates to distinguish CP-CRE and nonCP-CRE for epidemiological or infection control purposes. However, they were not currently recommended for routine clinical use. Thus, the First Affiliated Hospital of Chongqing Medical University Antibiotic Treatment Guidelines did not differentiate treatment recommendations for $\mathrm{CP}-\mathrm{CRE}$ and non-CP-CRE infections. As clinicians were unaware of carbapenemase gene results when selecting antibiotic therapy for CRE bacteremia, therapeutic regimens were mainly experience-directed. This study was novel in investigating the possible value of CP-CRE/non-CP-CRE stratification and CRE resistance mechanism determination in better managing CRE bacteremia by CAZ/AVI and ATM/AVI.

\section{Some Conclusions Of This Study Were Noteworthy}

First, the study explored the independent risk factors leading to the acquisition of CP-CRE bacteremia. Notably, besides 


\begin{tabular}{|c|c|c|c|c|c|c|c|c|c|c|c|}
\hline \multirow{2}{*}{ 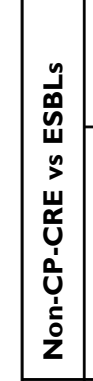 } & $\frac{\stackrel{0}{\bar{z}}}{\sum_{a}^{n}}$ & & $\begin{array}{ll}m \\
o \\
0\end{array}$ & & ঙํ & & 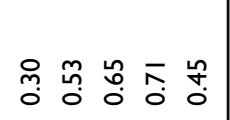 & & 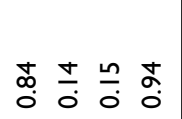 & & 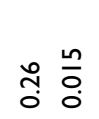 \\
\hline & 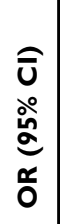 & & 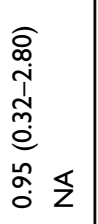 & & 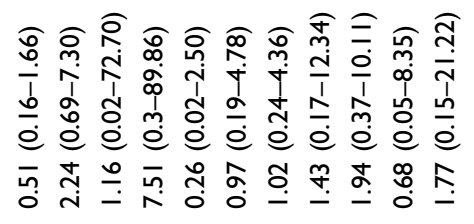 & & 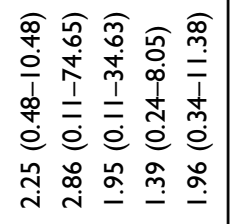 & & 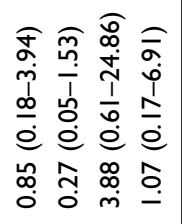 & & 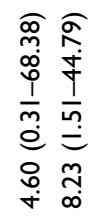 \\
\hline & $\frac{\mathscr{g}}{\frac{\partial}{\pi}}$ & & $\bar{\sigma}$. & & 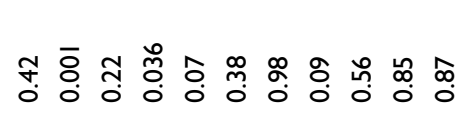 & & 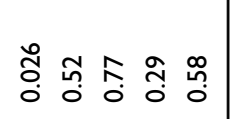 & & 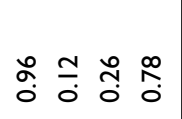 & & $\frac{7}{0}$ \\
\hline 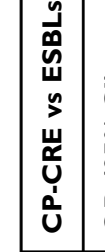 & 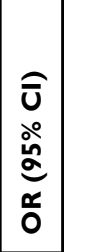 & & 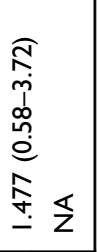 & & 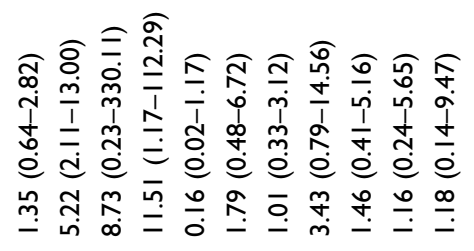 & & 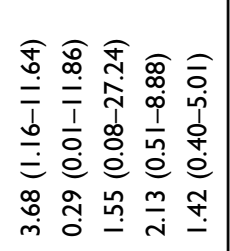 & & 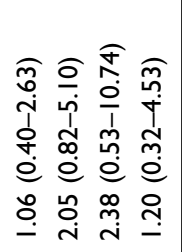 & & 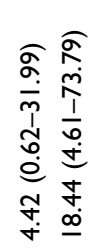 \\
\hline 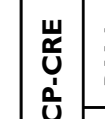 & 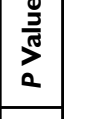 & & $\begin{array}{lll}0 & 0 \\
0 & 0 \\
\end{array}$ & & 瓷 贻 & & 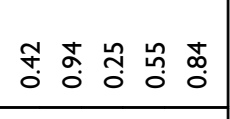 & & 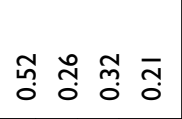 & & $\stackrel{0}{f} \stackrel{m}{0}$ \\
\hline 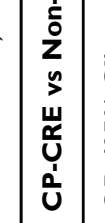 & 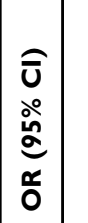 & & 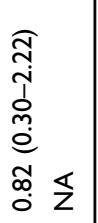 & & 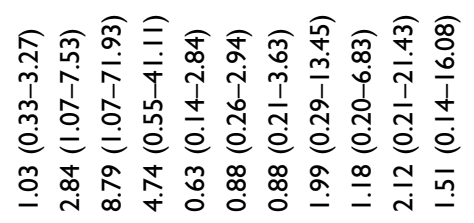 & & 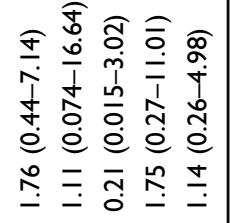 & & 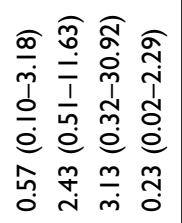 & & 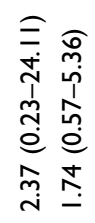 \\
\hline 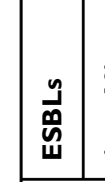 & $\begin{array}{ll} \\
\vdots \\
\text { "I } \\
\Xi\end{array}$ & & 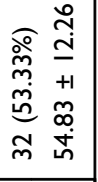 & & 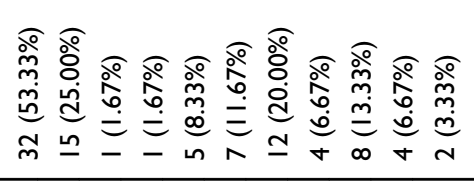 & & 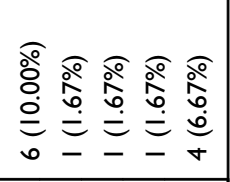 & & 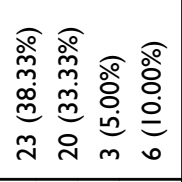 & & 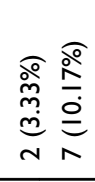 \\
\hline 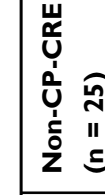 & & & 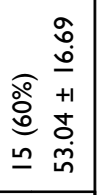 & & 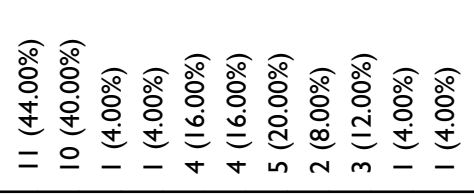 & & 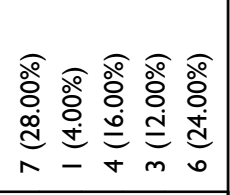 & & 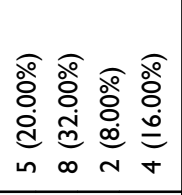 & & 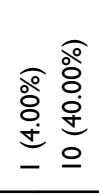 \\
\hline 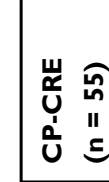 & & & 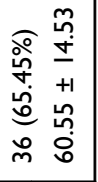 & & 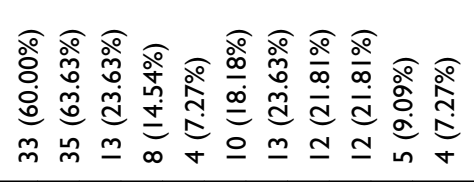 & & 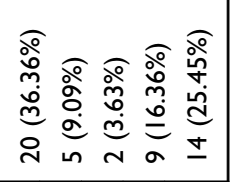 & & 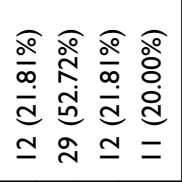 & & 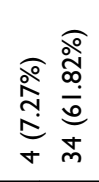 \\
\hline 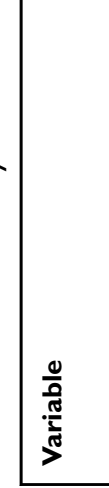 & & 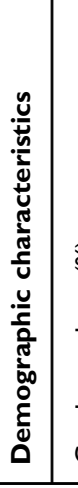 & 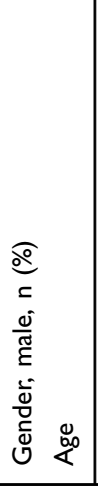 & 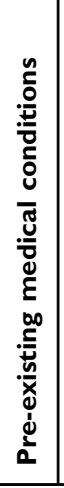 & 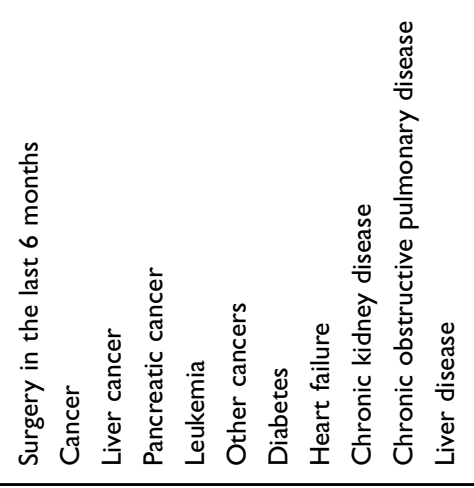 & 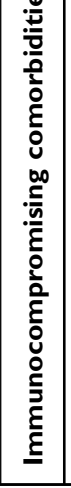 & 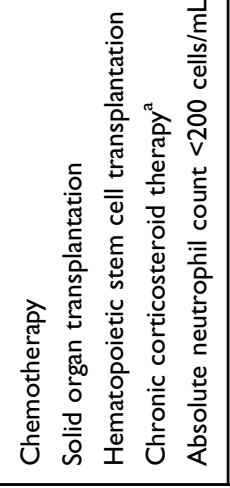 & 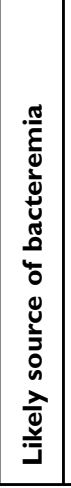 & 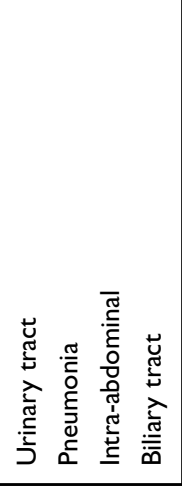 & 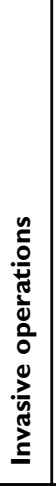 & 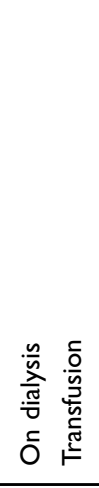 \\
\hline
\end{tabular}




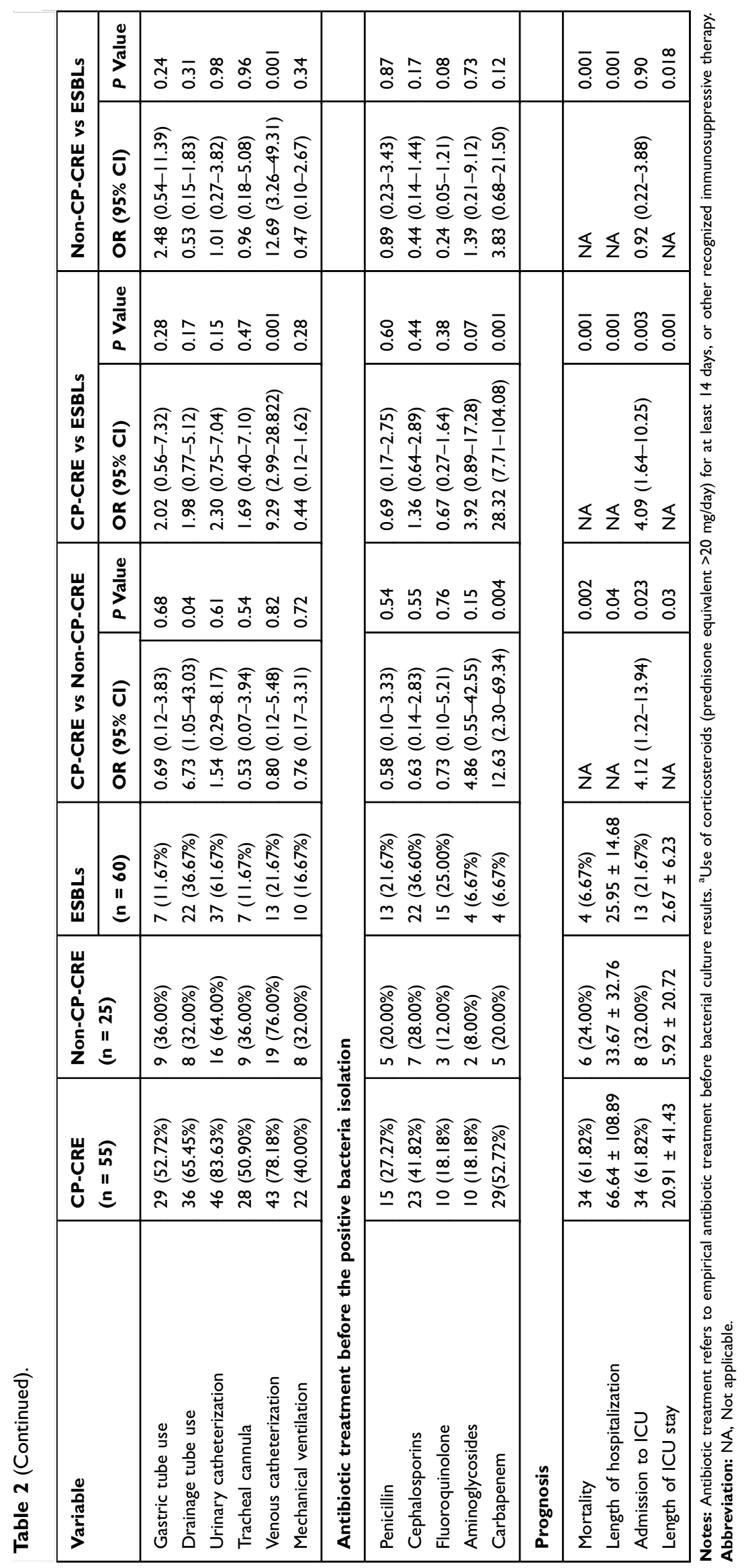


Table 3 Multivariate Analyses Of Risk Factors For The Isolation Of CP-CRE Group Versus Non-CP-CRE Group, CP-CRE Group Versus ESBL Group, And Non-CP-CRE Group Versus ESBL Group

\begin{tabular}{|l|l|l|l|}
\hline Variable & $\mathbf{9 5 \%}$ CI & OR & P Value \\
\hline CP-CRE vs non-CP-CRE & & & \\
Age & NA & NA & 0.04 \\
Admission to ICU & $0.59-7.55$ & 2.12 & 0.25 \\
Length of ICU stay & NA & NA & 0.03 \\
Cancer & $1.22-15.46$ & 4.35 & 0.023 \\
Liver cancer & $0.28-30.16$ & 2.91 & 0.37 \\
Use of drainage tube & $0.62-10.34$ & 2.23 & 0.19 \\
Previous carbapenem exposure & $1.91-30.77$ & 7.67 & 0.004 \\
\hline CP-CRE vs ESBLs & & & \\
Age & NA & NA & 0.01 \\
Admission to ICU & $0.10-2.20$ & 0.46 & 0.33 \\
Length of ICU stay & NA & NA & 0.02 \\
Transfusion & $3.15-93.72$ & 17.19 & $0.00 \mathrm{I}$ \\
Cancer & $3.56-71.37$ & 15.91 & $<0.001$ \\
Chemotherapy & $0.17-5.16$ & 0.94 & 0.94 \\
Venous catheterization & $1.82-35.90$ & 8.10 & 0.006 \\
Previous carbapenem exposure & $5.03-154.19$ & 27.86 & $0.00 \mathrm{I}$ \\
\hline Non-CP-CRE vs ESBLs & & & \\
Length of ICU stay & NA & NA & 0.018 \\
Transfusion & $0.32-4.78$ & 1.24 & 0.75 \\
Venous catheterization & $3.03-34.87$ & 10.29 & 0.001 \\
\hline
\end{tabular}

Abbreviation: NA, Not applicable.

old age, transfusion, longer ICU stay, and cancer, previous carbapenem exposure was identified as an independent risk factor for bacteremia CP-CRE acquisition, which was in agreement with a previous report. ${ }^{24}$
Second, CAZ/AVI was reported to be active in vitro against non-CP-CRE as well as $b l a_{\mathrm{KPC}}$ and $b l a_{\mathrm{OXA}-48^{-}}$-harboring CP-CRE isolates. ${ }^{25}$ Emerging clinical data demonstrated that CAZ/AVI treatment in CRE infections achieved clinical response rates that were superior to those attained with regimens including colistin or an aminoglycoside. ${ }^{26,27}$ At our center, in vitro CAZ/AVI resistance emerged in $29.31 \%$ of the CRE bacteremic isolates, which was congruent with a previous report by Shields who declared a $75 \%$ success rate for CAZ/AVI in treating CRE bacteremia. ${ }^{28}$ Moreover, consistent with previous reports, in vitro susceptibility testing showed that most CP-CRE bacteremia strains carrying the $b l a_{\mathrm{KPC}-2}$ gene were sensitive to CAZ/AVI. However, 2 out of 31 bla $_{\mathrm{KPC}-2}$-harboring strains (with ESBL overexpression and OMP loss) were revealed to be CAZ/AVI-resistant, mirroring the strains described in prior reports by Shields, who demonstrated a stepwise increase in CAZ/AVI MICs in KPC-2producing $K$. pneumoniae isolates co-harboring ESBLs and ompK36 porin gene mutations. ${ }^{29}$ Humphries thought that the extensive prior treatment with meropenem and cefepime might have provided the selective pressure required for a mutation to OmpK $36 .^{30}$ Giddins identified CAZ/AVI resistance in the newly emerging $K$. pneumoniae ST307 clonal background after only 12 days of CAZ/AVI exposure, with the induction of a $532 \mathrm{G}-\mathrm{T}$ mutation in the bla $_{\mathrm{KPC}-2}$ gene leading to a D179Y protein substitution as the putative initial mechanism of CAZ/AVI resistance. ${ }^{31}$ The characterization of some CAZ/ AVI-resistant Pseudomonas aeruginosa isolates demonstrated that the entry of CAZ/AVI into the periplasmic space depended on outer membrane permeability. ${ }^{32}$ Also, the patients infected with KPC2-producing CRE had no previous

Table 4 Distribution And Corresponding Carbapenem MIC Ranges For Strains With Different Resistance Determinants

\begin{tabular}{|c|c|c|c|c|}
\hline \multirow[t]{2}{*}{ Carbapenem Resistance Mechanisms } & \multirow[t]{2}{*}{ No. Of Isolates } & \multicolumn{3}{|c|}{ MIC Range $(\mu \mathrm{g} / \mathrm{mL})$} \\
\hline & & ETP & IMP & MEM \\
\hline Carbapenemase positive & 55 & & & \\
\hline NDM-I, ESBLs, OMP loss or deficiency & 10 & $8-256$ & $4-64$ & $4-128$ \\
\hline NDM-5, ESBLs, OMP loss or deficiency & 8 & $16-256$ & $8-256$ & $4-256$ \\
\hline KPC-2, OMP loss or deficiency & 34 & $4-128$ & $\mathrm{I}-128$ & $1-128$ \\
\hline IMP-4, ESBLs, OMP deficiency & I & 64 & 8 & 8 \\
\hline IMP-8, ESBLs, OMP loss & I & 8 & 8 & 8 \\
\hline KPC-2, IMP-4, ESBLs, OMP deficiency & I & 256 & 256 & 128 \\
\hline Carbapenemase negative & 26 & & & \\
\hline AmpC, ESBLs & I & 4 & 2 & 0.25 \\
\hline AmpC, OMP loss & I & 2 & 2 & 1 \\
\hline AmpC, ESBLs, OMP loss & I & 4 & 4 & 0.25 \\
\hline ESBLs, OMP loss or deficiency & 23 & $2-32$ & $0.25-16$ & $0.25-8$ \\
\hline
\end{tabular}




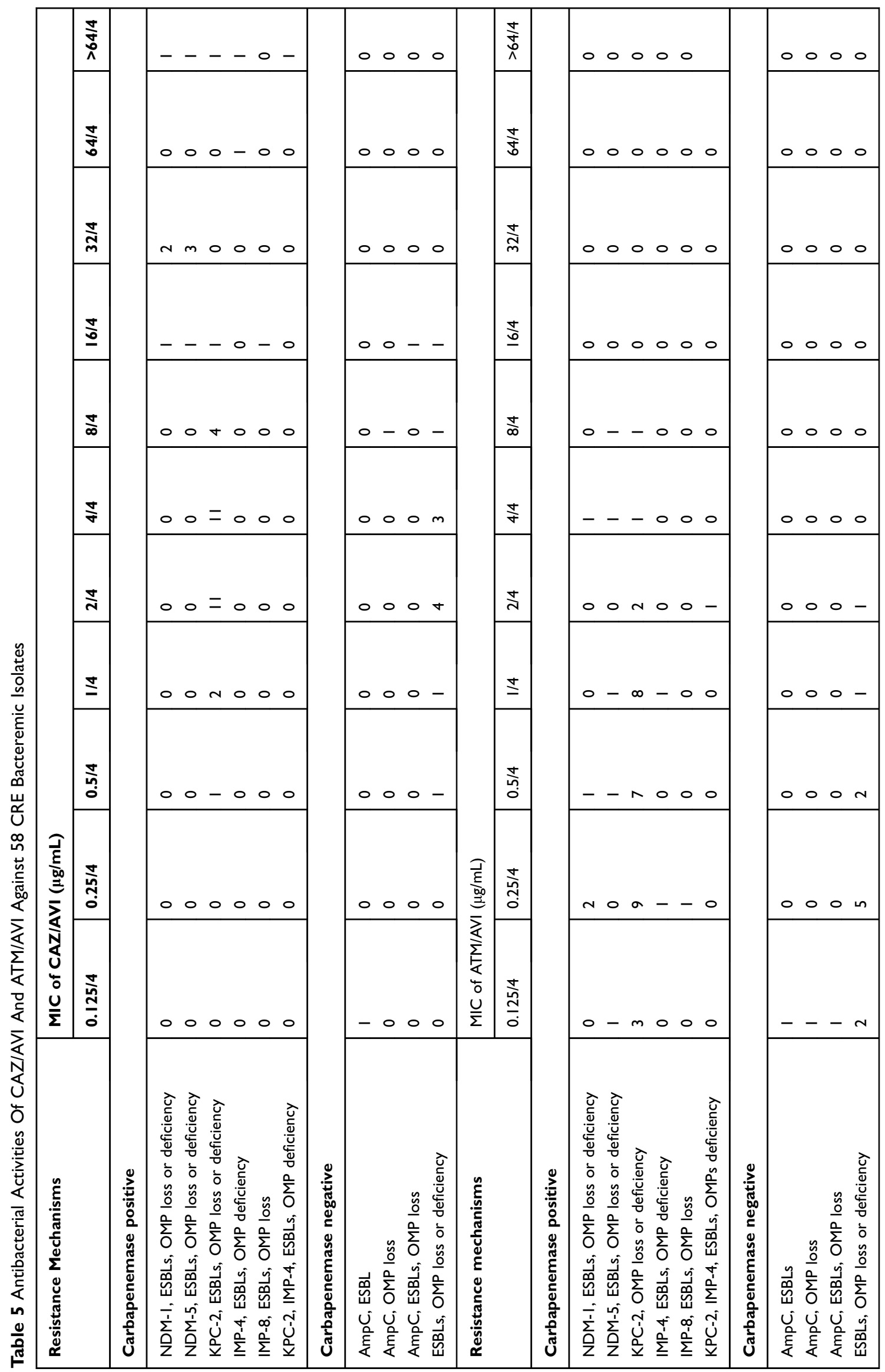


exposure to CAZ/AVI, but instead had frequent previous exposures to meropenem and cefepime. Hence, it was postulated that their resistance to CAZ/AVI might be also due to the lack of membrane porin proteins.

Third, ATM/AVI combination is currently in clinical development for treating serious infections caused by MBL-producing Enterobacteriaceae, ${ }^{19}$ with sporadically reported resistance. Fortunately, this study demonstrated that all the studied CRE bacteremic isolates, including both the CP-CRE and non-CP-CRE ones, were highly sensitive to ATM/AVI in vitro. Nevertheless, Alm already found a special NDM-producing $E$. coli isolate with decreased susceptibility to ATM/AVI caused by a fouramino-acid insertion in PBP3, leading to decreased binding. ${ }^{33}$ Hence, it is necessary to investigate whether ATM/AVI resistance or a stepwise increase in ATM/AVI MICs would be rapidly induced during ATM/AVI treatment, especially in the most notorious double-carbapenemase producers with porin loss/deficiency.

In general, with the advancement of individualized medicine, the selection and use of antibiotics should gradually be personalized and standardized. CP-CRE/non-CP-CRE bacteremia stratification and $\mathrm{CRE}$ resistance mechanism determination can better guide the clinical management of CRE bacteremia. For example, ATM/AVI can be prescribed to treat CP-CRE bacteremia for patients with high risks of contracting CP-CRE bacteremia, such as those with advanced age, transfusion, longer ICU stay, cancer, and previous exposure of carbapenems, while patients without risk factors can be treated with CAZ/AVI.

A limitation of the present study was that only the in vitro susceptibility tests of CAZ/AVI and ATM/AVI were performed. Animal experiments and prospective clinical trials are still needed to validate the risk factors and therapeutic recommendations based on CRE resistance mechanism stratification for CRE bacteremia. Furthermore, multi-center retrospective and prospective studies are still awaited to move from a proof of concept to practical applications of risk factors and management of CRE bacteremia guided by molecular resistance mechanism stratification. In addition, CP-CRE/ non-CP-CRE stratification and CRE resistance mechanism determination might also better guide the clinical management of CRE bacteremia using other novel antibiotics, such as meropenem/vaborbactam, imipenem/relebactam, and cefiderocol, which needs further investigation. Last but not the least, possible synergy of CAZ/AVI with meropenem, amikacin, aztreonam, colistin, or fosfomycin against the aforementioned CAZ/AVI-resistant isolates deserves further explorations.
Although much remains to be elucidated concerning the therapeutic recommendations for CRE bacteremia guided by the resistance mechanism stratification, this study laid an invaluable groundwork for future studies.

\section{Acknowledgments}

This study was supported in part by the National Natural Science Foundation of China (Grant No. 81772239 and 31500749), the Science and Technology Research Program of Chongqing Municipal Education Commission (Grant No. KJ1500235 and KJ1702022), and the Medical Research Program of Chongqing Health and Family Planning Commission (No. 2018MSXM009 and 2016MSXM001).

\section{Author Contributions}

HZ, SQN and SFH designed the study; HZ, QXL, SFH, and MLW performed the experiments; SJX and QXL analyzed data; HZ, SQN, and SFH wrote this manuscript. All authors contributed to data analysis, drafting or revising the article, gave final approval of the version to be published, and agree to be accountable for all aspects of the work.

\section{Disclosure}

The authors report no conflicts of interest in this work.

\section{References}

1. Durante-Mangoni E, Andini R, Zampino R. Management of carbapenem resistant Enterobacteriaceae infections. Clin Microbiol Infect. 2019;25(8):943-950. doi:10.1016/j.cmi.2019.04.013

2. Righi E, Peri AM, Harris PN, et al. Global prevalence of carbapenem resistance in neutropenic patients and association with mortality and carbapenem use: systematic review and meta-analysis. $J$ Antimicrob Chemother. 2017;72(3):668-677. doi:10.1093/jac/dkw459

3. Amit S, Mishali H, Kotlovsky T, Schwaber MJ, Carmeli Y. Bloodstream infections among carriers of carbapenem-resistant klebsiella pneumoniae: etiology, incidence and predictors. Clin Microbiol Infect. 2015;21(1):30-34. doi:10.1016/j.cmi.2014.08.001

4. Márió G. The concept of an ideal antibiotic: implications for drug design. Molecules. 2019;24(5):892. doi:10.3390/molecules24050892

5. Nordmann P, Dortet L, Poirel L. Carbapenem resistance in Enterobacteriaceae: here is the storm! Trends Mol Med. 2012;18 (5):263-272. doi:10.1016/j.molmed.2012.03.003

6. Lutgring JD, Limbago BM. The problem of carbapenemase-producing-carbapenem-resistant-Enterobacteriaceae detection. J Clin Microbiol. 2016;54(3):529-534. doi:10.1128/JCM.02771-15

7. Falagas ME, Lourida P, Poulikakos P, Rafailidis PI, Tansarli GS. Antibiotic treatment of infections due to carbapenem-resistant Enterobacteriaceae: systematic evaluation of the available evidence. Antimicrob Agents Chemother. 2014;58(2):654-663. doi:10.1128/ AAC.01222-13

8. Biedenbach DJ, Kazmierczak K, Bouchillon SK, Sahm DF, Bradford PA. In vitro activity of aztreonam-avibactam against a global collection of gram-negative pathogens from 2012 and 2013. Antimicrob Agents Chemother. 2015;59(7):4239-4248. doi:10.1128/ AAC.00206-15 
9. Falcone M, Paterson D. Spotlight on ceftazidime/avibactam: a new option for MDR gram-negative infections. J Antimicrob Chemother. 2016;71(10):2713-2722. doi:10.1093/jac/dkw239

10. Lomovskaya O, Sun D, Rubio-Aparicio D, et al. Vaborbactam: spectrum of beta-lactamase inhibition and impact of resistance mechanisms on activity in Enterobacteriaceae. Antimicrob Agents Chemother. 2017;61(11):e01443-e01517. doi:10.1128/AAC.0 1443-17

11. Haidar G, Clancy CJ, Chen L, et al. Identifying spectra of activity and therapeutic niches for ceftazidime-avibactam and imipenem-relebactam against carbapenem-resistant Enterobacteriaceae. Antimicrob Agents Chemother. 2017;61(9):e00642-e00717. doi:10.1128/AAC.00642-17

12. Monogue ML, Thabit AK, Hamada Y, Nicolau DP. Antibacterial efficacy of eravacycline in vivo against gram-positive and gramnegative organisms. Antimicrob Agents Chemother. 2016;60 (8):5001-5005. doi:10.1128/AAC.00366-16

13. Denervaud-Tendon V, Poirel L, Connolly LE, Krause KM, Nordmann P. Plazomicin activity against polymyxin-resistant Enterobacteriaceae, including MCR-1-producing isolates. J Antimicrob Chemother. 2017;72(10):2787-2791. doi:10.1093/jac/ dkx239

14. Hackel MA, Tsuji M, Yamano Y, Echols R, Karlowsky JA, Sahm DF. In vitro activity of the siderophore cephalosporin, cefiderocol, against carbapenem-nonsusceptible and multidrug-resistant isolates of gram-negative bacilli collected worldwide in 2014 to 2016. Antimicrob Agents Chemother. 2018;62(2):e01968-17. doi:10.1128/AAC.01968-17

15. Tamma PD, Goodman KE, Harris AD, et al. Comparing the outcomes of patients with carbapenemase-producing and non-carbapenemaseproducing carbapenem-resistant Enterobacteriaceae bacteremia. Clin Infect Dis. 2017;64(3):257-264. doi:10.1093/cid/ciw741

16. Wong D, van Duin D. Novel beta-lactamase inhibitors: unlocking their potential in therapy. Drugs. 2017;77(6):615-628. doi:10.1007/ s40265-017-0725-1

17. Hsueh SC, Lee YJ, Huang YT, Liao CH, Tsuji M, Hsueh PR. In vitro activities of cefiderocol, ceftolozane/ tazobactam, ceftazidime/avibactam and other comparative drugs against imipenem-resistant Pseudomonas aeruginosa and Acinetobacter baumannii, and Stenotrophomonas maltophilia, all associated with bloodstream infections in Taiwan. J Antimicrob Chemother. 2019;74(2):380-386. doi:10.1093/jac/dky425

18. Emeraud C, Escaut L, Boucly A, et al. Aztreonam plus clavulanate, tazobactam, or avibactam for treatment of infections caused by metallobeta-lactamase-producing gram-negative bacteria. Antimicrob Agents Chemother. 2019;63(5):e00010-19. doi:10.1128/AAC.00010-19

19. Hobson CA, Bonacorsi S, Fahd M, et al. Successful treatment of bacteremia due to NDM-1-producing Morganella morganii with aztreonam and ceftazidime-avibactam combination in a pediatric patient with hematologic malignancy. Antimicrob Agents Chemother. 2019;63(2). doi:10.1128/AAC.00779-19

20. Das S, Li J, Riccobene T, et al. Dose selection and validation for ceftazidime-avibactam in adults with complicated intra-abdominal infections, complicated urinary tract infections, and nosocomial pneumonia. Antimicrob Agents Chemother. 2019;63(4). doi:10.1128/ AAC.00779-19
21. Wang Q, Wang X, Wang J, et al. Phenotypic and genotypic characterization of carbapenem-resistant Enterobacteriaceae: data from a longitudinal large-scale CRE study in China (2012-2016). Clin Infect Dis. 2018;67(suppl_2):S196-S205. doi:10.1093/cid/ciy660

22. Tian X, Sun S, Jia X, Zou H, Li S, Zhang L. Epidemiology of and risk factors for infection with extended-spectrum beta-lactamase-producing carbapenem-resistant Enterobacteriaceae: results of a double case-control study. Infect Drug Resist. 2018;11:1339-1346. doi:10.2147/IDR.S173456

23. French CE, Coope C, Conway L, et al. Control of carbapenemase-producing Enterobacteriaceae outbreaks in acute settings: an evidence review. $J$ Hosp Infect. 2017;95(1):3-45. doi:10.1016/j.jhin.2016.10.006

24. Li X, Ye H. Clinical and mortality risk factors in bloodstream infections with carbapenem-resistant Enterobacteriaceae. Can J Infect Dis Med Microbiol. 2017;2017:6212910. doi:10.1155/2017/6212910

25. Sousa A, Perez-Rodriguez MT, Soto A, et al. Effectiveness of ceftazidime/avibactam as salvage therapy for treatment of infections due to OXA-48 carbapenemase-producing Enterobacteriaceae. J Antimicrob Chemother. 2018;73(11):3170-3175. doi:10.1093/jac/dky295

26. Shirley M. Ceftazidime-avibactam: a review in the treatment of serious gram-negative bacterial infections. Drugs. 2018;78(6):675692. doi:10.1007/s40265-018-0902-x

27. Marshall S, Hujer AM, Rojas LJ, et al. Can ceftazidime-avibactam and aztreonam overcome beta-lactam resistance conferred by metallo-beta-lactamases in Enterobacteriaceae? Antimicrob Agents Chemother. 2017;61(4):e02243-16. doi:10.1128/AAC.02243-16

28. Shields RK, Nguyen MH, Chen L, Press EG, Kreiswirth BN, Clancy CJ. Pneumonia and renal replacement therapy are risk factors for ceftazidimeavibactam treatment failures and resistance among patients with carbapenem-resistant Enterobacteriaceae infections. Antimicrob Agents Chemother. 2018;62:5. doi:10.1128/AAC.02497-17

29. Shen Z, Ding B, Ye M, et al. High ceftazidime hydrolysis activity and porin OmpK35 deficiency contribute to the decreased susceptibility to ceftazidime/avibactam in KPC-producing Klebsiella pneumoniae. J Antimicrob Chemother. 2017;72(7):1930-1936. doi:10.1093/jac/dkx066

30. Nelson K, Hemarajata P, Sun D, et al. Resistance to ceftazidime-avibactam is due to transposition of KPC in a porin-deficient strain of Klebsiella pneumoniae with increased efflux activity. Antimicrob Agents Chemother. 2017;61(10):e00989-17. doi:10.1128/AAC.00989-17

31. Giddins MJ, Macesic N, Annavajhala MK, et al. Successive emergence of ceftazidime-avibactam resistance through distinct genomic adaptations in blaKPC-2-harboring Klebsiella pneumoniae sequence type 307 isolates. Antimicrob Agents Chemother. 2018;62(3):e0210117. doi:10.1128/AAC.02101-17

32. Wi YM, Greenwood-Quaintance KE, Schuetz AN, et al. Activity of ceftolozane-tazobactam against carbapenem-resistant, non-carbapenemase-producing Pseudomonas aeruginosa and associated resistance mechanisms. Antimicrob Agents Chemother. 2018;62(1): e01970-17.

33. Alm RA, Johnstone MR, Lahiri SD. Characterization of Escherichia coli NDM isolates with decreased susceptibility to aztreonam/avibactam: role of a novel insertion in PBP3. J Antimicrob Chemother. 2015;70(5):1420-1428. doi:10.1093/jac/dku568

Infection and Drug Resistance

Dovepress

\section{Publish your work in this journal}

Infection and Drug Resistance is an international, peer-reviewed openaccess journal that focuses on the optimal treatment of infection (bacterial, fungal and viral) and the development and institution of preventive strategies to minimize the development and spread of resistance. The journal is specifically concerned with the epidemiology of

antibiotic resistance and the mechanisms of resistance development and diffusion in both hospitals and the community. The manuscript management system is completely online and includes a very quick and fair peerreview system, which is all easy to use. Visit http://www.dovepress.com/ testimonials.php to read real quotes from published authors.

Submit your manuscript here: https://www.dovepress.com/infection-and-drug-resistance-journa 